\title{
The turbomolecular pump in molecular state
}

\author{
A G Antoniou and S E Valamontes, Cosmofarm Ltd, P.O. Box 42, 20100 Korinthos, Greece \\ and \\ C N Panos, Department of Physics, Technological and Educational Institute of Piraeus, Thivon 250, \\ 12244 Aegaleo, Greece
}

and

E S Valamontes, Department of Physics, Technological and Educational Institute of Chalkis, 34400 Psahna-Euboea, Greece

received 15 November 1994

\begin{abstract}
A new approach and successful method of studying the turbomolecular vacuum pump. Gaede's equations and Becker's treatment lead to two equations $\mathrm{k}=\mathrm{f}_{1}\left(\mathrm{u}_{0}, \mathrm{~s}, \mathrm{~g}\right)$ and $\mathrm{S}=\mathrm{f}_{2}\left(\mathrm{u}_{0}, \mathrm{~s}, \mathrm{~g}\right)$, which connect the compression ratio $\mathrm{k}$ and the pumping speed $\mathrm{S}$ with the rotational speed $\mathrm{u}_{0}$, the tangent $\mathrm{s}$ of the blade's angle and the geometry g of such a pump. The design of a turbomolecular vacuum pump is then possible through its geometry and its losses.
\end{abstract}

\section{Introduction}

Even if the invention of the turbomolecular vacuum pump belongs to Becker, ${ }^{1}$ the theoretical modeling was made first by Kruger and Shapiro. ${ }^{2}$ They calculated the probabilities of molecules passing through the blades of a single disc pump, from the side of low pressure to the side of high pressure and inversely. They calculated the compression and the pumping speed of a disc, as a function of the angle of the blades, the rotational speed, and the nature of the gas. In these calculations, the peripheral losses of the system were considered negligible.

The theoretical treatment of Becker ${ }^{3}$ is based on Gaede's ${ }^{4}$ equation which refers to the distribution of pressures in cross sectional tubes and helps calculate the compression and the pumping speed for one stage of a turbomolecular pump, as a function of the rotational speed, the coefficient of external viscosity which depends on the nature of the gas, the geometry of the stage, and all the peripheral losses.

Hence, there are two ways to consider the problem each with advantages and disadvantages. Since then, many theoretical works have been published, the majority of which follow the method of Kruger and Shapiro.

Our approach is based on the work of Gaede and Becker and its aim is to obtain two simple equations. One for compression $k$ $\left[k=f_{1}\left(u_{0}, g, s, \theta\right)\right]$ and the other for the pumping speed $S\left[S=f_{2}\right.$ $\left.\left(u_{0}, g, s, \theta\right)\right]$ of a turbomolecular vacuum pump, both as a function of the rotational speed of the discs $\left(u_{0}\right)$, the geometrical characteristics of the pump $(g)$, the tangent of the angle $\varphi$ of the blades $(s)$, and the external viscosity $(\theta)$, which depends on the nature of the gas and all the peripheral losses. In this approach there are two goals that can be achieved. The first is the choice of the angle $\varphi$ for a given construction, and the second is the reduction of the problem for turbomolecular pump to that of multi-grove molecular pumps in the molecular state.

\section{Theory}

Becker found experimentally ${ }^{5}$ that, when in a turbomolecular vacuum pump, shown in Fig. 1, there are optically opaque discs in the direction of the rotational axis, the pumping speed $S$ and the compression ratio $k$ can be improved substantially. This fact makes possible the design of a pump with infinite stages, according to Fig. 2(a). In this figure we mark with bold lines, the rotor and stator blades, and with the bold dotted lines, the mirror images of the rotor blades with respect to the optical opaqueness axis. Also, in the same figure, we take into consideration the case where the stator and the mirror images of the rotor blades to be ordered in such a way as to form parallel helices, which as we shall see later, improve the performance of the turbomolecular pump.

The following notations are used in Fig. 2(a):

$v$ is the number of the disc blades,

$\varphi$ is the angle of the blades $\left(^{\circ}\right)$,

$r$ is the mean radius of the blades (m),

$w$ is the thickness of the optical opaqueness (m),

$b^{\prime}$ is the length of the blades (m),

$h$ is the height of pumping channel (between blades) (m),

$L$ is the front blade distance (m),

$L^{\prime}$ is the blade thickness parallel to the rotational axis (m),

$b^{\prime \prime}$ is the disc thickness (m),

$h^{\prime \prime}$ is the distance between the discs ( $\mathrm{m}$ ) and

$h^{\prime}$ is the distance between rotor and stator $(\mathrm{m})$, 


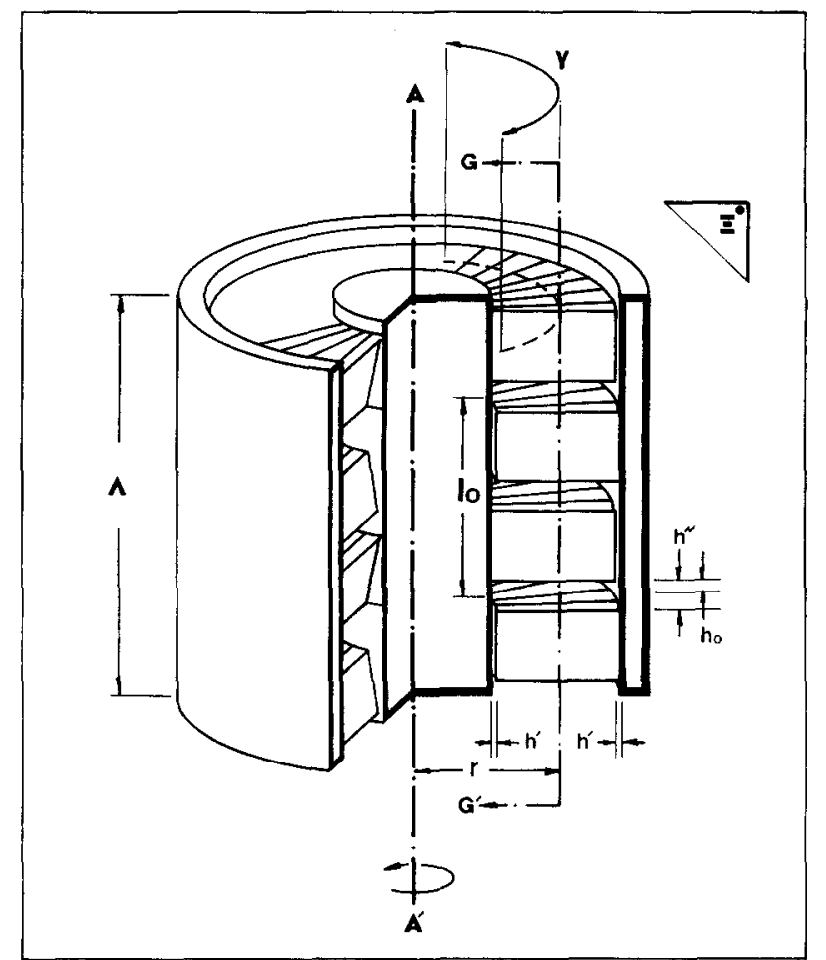

Figure 1. Cross sectional area of a three-dimensional turbomolecular vacuum pump.

When the rotor is rotating with a frequency $n\left[\mathrm{sec}^{-1}\right]$ it produces, in first approximation, a relative velocity $2 \pi r n(\cos \varphi)$, responsible for the pumping action. This result permits one to replace the rotating discs by an ideal system (drawn with bold dotted lines) moving in the $x$-direction with velocity $u=2 \pi r n(\cos \varphi)$, producing the same result (Fig. 2(a)).

Figure 2(b) shows the details of Fig. 2(a) around the points $B_{1}, B_{0}$ and $B_{2}$. It gives us the possibility to treat in a similar way to Gaede the problem of the turbomolecular vacuum pump calculations, as we have already done in the multi-groove helicoid pump in the molecular state. ${ }^{6}$

Bearing in mind the losses during the rotation of the rotor and its status of dynamic equilibrium, we can refer to three different gas quantities that backstream: The first gas quantity which consists of a loss between the two discs in the channel of height $h_{0}$ and width $b^{\prime}$, due to the motion of only one of the discs, is

$\mathrm{d} Q_{1}=\frac{u_{1}+u_{2}}{2} b^{\prime} h_{0} p$

where

$h_{0}=\frac{h^{\prime 2}}{2\left(d^{\prime \prime}+h^{\prime \prime}\right)}$

is the effective height of Becker's, ${ }^{7}$ and $p$ is the pressure. This gas quantity per unit length becomes

$\mathrm{d} Q_{1}=\frac{u_{1}+u_{2}}{2} b^{\prime} h_{0} p \frac{\mathrm{d} x}{(l / 2)}$.

The second gas quantity which consists of a loss through the parallel to the rotational axis channel, of width $\mathrm{d} x_{1}$, height $h^{\prime}$
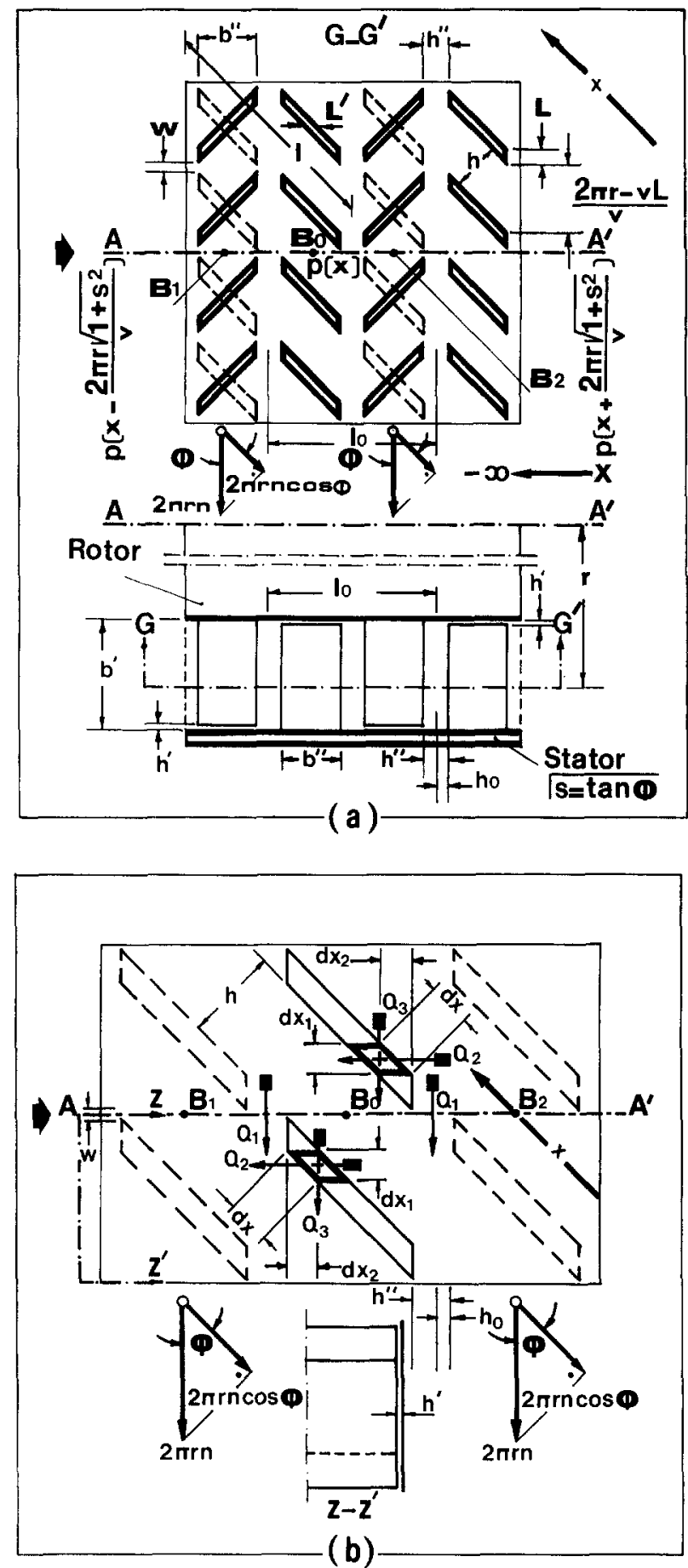

Figure 2. (a) Down: Cross-section of Fig. 1, with respect to $\Xi$-plane. Up : An envelope of the same cross section with respect to $\operatorname{arc} \gamma$ (b) Detailed schematic presentation of the region hetween points $B_{1}, B_{0}$ and $B_{2}$, as shown in Fig. 2(a).

and length $L^{\prime}$, due to the pressure difference $\Delta p$ at the ends of $L^{\prime}$, is

$\mathrm{d} Q_{2}=\frac{h^{\prime 2}}{2 L^{\prime} \theta} \cdot \frac{\Delta p}{\alpha} \mathrm{d} x_{1}$

where $a$ is the ratio of the pressure difference between the points $B_{0}$ and $B_{2}$ to the pressure difference at the ends of $L^{\prime}$ or equivalently the ratio of the distances between the points $B_{0}$ and $B_{2}$ 
to the length $L^{\prime}$, and $\theta[\theta=\theta(T, m), T=$ absolute temperature, $m=$ mass of a molecule] is the coefficient of external viscosity which defines the friction between the gas and the internal wall of the pump. (Becker refers to $\theta$ as coefficient of external friction.)

Finally, the third gas quantity which consists of a flow perpendicular to the rotational axis through a channel of width $\mathrm{d} x_{2}$ and height $h^{r}$, due to the cylindrical rotation of the surfaces of the discs, is

$\mathrm{d} Q_{3}=\frac{u_{1}+u_{2}}{2} h^{\prime} p \mathrm{~d} x_{2}$

From the geometry of Figs $2(a)$ and (b) we have :

$\mathrm{d} x_{1}=(\cos \phi) \mathrm{d} x, \quad \mathrm{~d} x_{2}=(\sin \phi) \mathrm{d} x$

$u_{1}=2 \pi r n, \quad u_{2}=0$

$L^{\prime}=L \cdot \tan \phi, \quad h=\frac{(2 \pi r-v L)}{v} \sin \phi$

By using eqns (4)-(6) and replacing $\tan \varphi$ by $s(\tan \varphi=s)$, eqns (2) and (3) become :

$\mathrm{d} Q_{1}=\frac{2 \pi r n b^{\prime} h_{0}}{l} p \mathrm{~d} x$

$\mathrm{d} Q_{2}=\frac{h^{\prime 2}}{\alpha 2 \theta L s \sqrt{1+s^{2}}} \Delta p \mathrm{~d} x$

$\mathrm{d} Q_{3}=\frac{\pi r n h^{\prime} s}{\sqrt{1+s^{2}}} p \mathrm{~d} x$.

Considering now the three arranged points $B_{1}, B_{0}, B_{2}$ we have that, if at point $B_{0}$ of the groove the pressure is $p=p(x)$, then at point $B_{2}$ the pressure will be $p=p\left(x+2 \pi r \sqrt{1+s^{2}} / v\right)$, while at point $B_{1}$ the pressure will be $p=p\left(x-2 \pi r \sqrt{1+s^{2}} / v\right.$ ), (where $v$ represents also the number of grooves). Thus from eqns (7)-(9) it comes out that the elementary gas quantity (of the gas to be pumped) that backstreams from higher to lower pressure and consists of the loss of the system for length $\mathrm{d} x$ is $\mathrm{d} Q=\mathrm{d} Q_{1}+\mathrm{d} Q_{2}+\mathrm{d} Q_{3}$

$$
\begin{aligned}
Q & =\left\{\frac { h ^ { \prime 2 } } { \alpha 2 \theta L s \sqrt { 1 + s ^ { 2 } } } \left[p\left(x+\frac{2 \pi r \sqrt{1+s^{2}}}{v}\right)\right.\right. \\
& \left.+p\left(x-\frac{2 \pi r \sqrt{1+s^{2}}}{v}\right)-2 p(x)\right]+\left[\frac{\pi r n h^{\prime} s}{2 \sqrt{1+s^{2}}}+\frac{\pi r n b^{\prime} h_{0}}{l}\right] \\
& \left.+\left[p\left(x+\frac{2 \pi r \sqrt{1+s^{2}}}{v}\right)-p\left(x-\frac{2 \pi r \sqrt{1+s^{2}}}{v}\right)\right]\right\} \mathrm{d} x
\end{aligned}
$$

But because the pressure as a function of $x$, length of the groove, is given by

$p=p_{\mathrm{s}} \exp (m x)$

where $m$ is a constant ${ }^{6}$ and $p_{\mathrm{s}}$ the separation pressure, ${ }^{6}$ eqn (10) becomes

$$
\begin{aligned}
& \mathrm{d} Q=p_{\mathrm{s}}\left\{\frac { h ^ { 2 } } { \alpha 2 \theta L s \sqrt { 1 + s ^ { 2 } } } \left[\exp \left(\frac{m 2 \pi r \sqrt{1+s^{2}}}{v}\right)\right.\right. \\
& \left.+\exp \left(-\frac{m 2 \pi r \sqrt{1+s^{2}}}{v}\right)-2\right]+\left(\frac{\pi r n h^{\prime} s}{2 \sqrt{1+s^{2}}}+\frac{\pi r n b^{\prime} h_{0}}{l}\right) \\
& \left.\cdot\left[\exp \left(\frac{m 2 \pi r \sqrt{1+s^{2}}}{v}\right)-\exp \left(\frac{m 2 \pi r \sqrt{1+s^{2}}}{v}\right)\right]\right\} \exp (m x) \mathrm{d} x .
\end{aligned}
$$

After intcgration for $x$, wc have

$$
\begin{aligned}
Q & =p_{\mathrm{s}}\left\{\frac { h ^ { \prime 2 } } { \alpha 2 \theta L s \sqrt { 1 + s ^ { 2 } } } \left[\exp \left(\frac{m 2 \pi r \sqrt{1+s^{2}}}{v}\right)\right.\right. \\
& \left.\left.+\exp \left(-\frac{m 2 \pi r \sqrt{1+s^{2}}}{v}\right)-2\right)\right]+\left(\frac{\pi r n h^{\prime} s}{2 \sqrt{1+s^{2}}}+\frac{\pi r m b^{\prime} h_{0}}{l}\right) \\
& \left.\cdot\left[\exp \left(\frac{m 2 \pi r \sqrt{1+s^{2}}}{v}\right)-\exp \left(\frac{m 2 \pi r \sqrt{1+s^{2}}}{v}\right)\right]\right\} \frac{\operatorname{cxp}(m x)}{m} .
\end{aligned}
$$

During pumping of quantity $Q$, Gaede's ${ }^{4}$ equation will also be valid

$Q=\frac{u_{1}+u_{2}}{2} b h p-\frac{b h^{2}}{2 \theta} \frac{\mathrm{d} p}{\mathrm{~d} x}$.

Equation (14), in combination with eqns (4)-(6) and (11) takes the form

$Q=p_{s}\left[\frac{\pi r n b^{\prime}(2 \pi r-v L) s}{v\left(1+s^{2}\right)}-\frac{b^{\prime}(2 \pi r-v L)^{2} s^{2} m}{2 \theta v^{2}\left(1+s^{2}\right)}\right] \exp (m x)$

From eqns (13) and (15) and after the simplifications results the mathematical identity

$$
\begin{gathered}
\frac{h^{\prime 2}}{\alpha 2 \theta L s \sqrt{1+s^{2}}}\left[\exp \left(\frac{m 2 \pi r \sqrt{1+s^{2}}}{v}\right)\right. \\
\left.+\exp \left(-\frac{m 2 \pi r \sqrt{1+s^{2}}}{v}\right)-2\right]+\left(\frac{\pi r n h^{\prime} s}{2 \sqrt{1+s^{2}}}+\frac{\pi r n b^{\prime} h_{0}}{l}\right) \\
\cdot\left[\exp \left(\frac{m 2 \pi r \sqrt{1+s^{2}}}{v}\right)-\exp \left(\frac{m 2 \pi r \sqrt{1+s^{2}}}{v}\right)\right] \\
=\frac{\pi r n b^{\prime}(2 \pi r-v L) s m}{v\left(1+s^{2}\right)} b^{\prime}(2 \pi r-v L)^{2} s^{2} m^{2} \\
2 \theta v^{2}\left(1+s^{2}\right)
\end{gathered}
$$

\section{3. $k$ calculations}

If at any point $x$ of the groove the pressure is $p=p_{\mathrm{s}} \exp (m x)$, then at the point $x+2 \pi r \sqrt{1+s^{2}} / v$ the pressure will be 
$p=p_{\mathrm{s}} \exp \left[m\left(x+2 \pi r \frac{\sqrt{1+s^{2}}}{\nu}\right)\right]$

and the compression of the turbomolecular pump will also be

$k=\exp \left(m 2 \pi r \frac{\sqrt{1+s^{2}}}{v}\right)$

Thus $m$, the rate of the exponential variation of pressure, across the length of the groove will be

$m=\frac{v}{2 \pi r \sqrt{1+s^{2}}} \ln k$

Replacing $m$ from eqn (18) and $\exp \left[m 2 \pi r\left(1+s^{2}\right)^{1 / 2} / v\right]$ from eqn (17), into mathematical identity (16), we have

$$
\begin{aligned}
& \frac{h^{\prime 2}(2 \pi r)^{2}}{\alpha L b^{\prime}(2 \pi r-v L)^{2}}\left(\frac{\sqrt{1+s^{2}}}{s}\right)^{3} \frac{(k-1)^{2}}{k}+\left(\frac{h^{\prime}}{2} \frac{s}{\sqrt{1+s^{2}}}+\frac{b^{\prime} h_{0}}{l}\right) \\
& \times \frac{(2 \pi r)^{3} \theta n}{b^{\prime}(2 \pi r-v L)^{2}}\left(\frac{1+s^{2}}{s}\right)^{2} \frac{\left(k^{2}-1\right)}{k} \\
& -\frac{n \theta(2 \pi r)^{2}}{(2 \pi r-v L)} \frac{\sqrt{1+s^{2}}}{s} \ln k+\ln ^{2} k=0 .
\end{aligned}
$$

Also from Fig. 2(a) it can be seen that the following equations are valid:

$\alpha=\frac{2 \pi r}{v L}=\frac{B_{2} B_{0}}{L^{\prime}}, \quad l=\frac{4 \pi r}{v} \sqrt{1+s^{2}}$

$h_{0}=\frac{h^{\prime \prime 2}}{2\left(b^{\prime \prime}+h^{\prime}\right)} \cong \frac{h^{\prime \prime 2} v}{(4 \pi r) s}$.

Then eqn (19) becomes :

$$
\begin{aligned}
& \frac{(2 \pi r) h^{\prime 2} v}{b^{\prime}(2 \pi r-v L)^{2}}\left(\frac{\sqrt{1+s^{2}}}{s}\right)^{3} \frac{(k-1)^{2}}{k}+\left(\frac{h^{\prime}}{2} s^{2}+\frac{b^{\prime} h^{\prime 2} v^{2}}{(4 \pi r)^{2}}\right) \\
& \cdot \frac{(2 \pi r)^{3} \theta n}{b^{\prime}(2 \pi r-v L)^{2}}\left(\frac{\sqrt{1+s^{2}}}{s}\right)^{3} \frac{\left(k^{2}-1\right)}{k} \\
& -\frac{(2 \pi r)^{2} n \theta}{(2 \pi r-v L)} \frac{\sqrt{1+s^{2}}}{s} \ln k+\ln ^{2} k=0
\end{aligned}
$$

which is almost similar to that of Gaede's equation ${ }^{4}$ which refers to the molecular single groove pumps when they operate in the molecular state. The solution of equation 22 (which must give values for $k>1$ ) depends mainly on $h^{\prime}$ and $h^{\prime \prime}$.

\section{4. $S$ calculation}

We have mentioned that during the pumping of quantity $Q$ from each groove, eqn (14) is valid and the pumping speed $S=Q / p$ will be

$S=\frac{u_{1}+u_{2}}{2} b h-\frac{b h^{2}}{2 \theta p} \frac{\mathrm{d} p}{\mathrm{~d} x}$

which in the case of the first stage at the inlet of the pump, takes the form
$S=\frac{u_{1}+u_{2}}{2} h h-\frac{b h^{2}}{2 \theta l} \frac{p_{1}-p_{2}}{p_{1}}$

where $p_{1}$ and $p_{2}$ are the corresponding pressures in both sides of the stage. Because $p_{1}=k^{2} p^{2}$ and $b=b^{\prime}$ and eqns (5), (6) and (20) are valid, finally the pumping speed for the $v$ grooves of the pump will be

$$
\begin{aligned}
S= & b^{\prime}(2 \pi r-v L) \frac{s}{\sqrt{1+s^{2}}} \\
& \times\left[\frac{\pi r n}{\sqrt{1+s^{2}}}-\frac{(2 \pi r}{(8 \pi r) \theta} \frac{v L)}{1+s^{2}} \frac{s}{k^{2}}\right]
\end{aligned}
$$

Therefore, in the manner that we have considered the problem of a turbomolecular vacuum pump, we finally obtain the equations (22) and (25) where the compression ratio and the pumping speed are functions of the geometrical parameters $h^{\prime}, h^{\prime \prime}, b^{\prime}, r$ and the number of rotations $n$.

\section{Numerical application and determination of angle $\varphi$}

If now we want to determine an angle $\varphi$ as a function of $k$ and $S$, for which the pump will have the optimum performance, we have to restrict ourselves to a specific pump with a specific rotational speed as in the following example.

A turbomolecular pump with the following characteristic dimensions is a common device:

$$
\begin{array}{ll}
\text { number of rotations } & \left.n=500\left(\mathrm{sec}^{-1}\right]\right), \\
\text { mean radius } & r=75 \times 10^{-3}(\mathrm{~m}), \\
\text { distance between the discs } & h^{\prime \prime}=0,5 \times 10^{-3}(\mathrm{~m}), \\
\text { gap loss } & h^{\prime}=0,25 \times 10^{-3}(\mathrm{~m}), \\
\text { number of blades in each disc } & v=48 \\
\text { length of the blades } & b^{\prime}=30 \times 10^{-3}(\mathrm{~m}), \text { and } \\
\text { front width of the blade } & L=4,3 \times 10^{-3}(\mathrm{~m}) .
\end{array}
$$

Supposing that this pump has an infinite length then, we apply equations (22) and (25) to nitrogen, helium and hydrogen :

For nitrogen $\left(\theta=1.58 \times 10^{-3}\left(\mathrm{sec} \cdot \mathrm{m}^{-1}\right)\right)$

$$
\begin{aligned}
& 6.7285 \times 10^{-4}\left(\frac{\sqrt{1+s^{2}}}{s}\right)^{3} \frac{(k-1)^{2}}{k} \\
& +\left(1.25 \times 10^{-4} \times s^{2}+1.9457 \times 10^{-6}\right) 39.299\left(\frac{\sqrt{1+s^{2}}}{s}\right)^{3} ; \\
& \times \frac{\left(k^{2}-1\right)}{k}-0.663 \frac{\sqrt{1+s^{2}}}{s} \ln k+\ln ^{2} k=0 \\
& S=7.938 \times 10^{-3} \frac{s}{\sqrt{1+s^{2}}} \\
& \quad \times\left[\frac{117.809}{\sqrt{1+s^{2}}}-88.7974 \frac{s}{1+s^{2}} \cdot \frac{\left(k^{2}-1\right)}{k^{2}}\right] .
\end{aligned}
$$

For helium $\left(\theta=0.597 \times 10^{-3}\left(\mathrm{sec} \cdot \mathrm{m}^{-1}\right)\right)$ 


$$
\begin{aligned}
& 6.7285 \times 10^{-4}\left(\frac{\sqrt{1+s^{2}}}{s}\right)^{3} \frac{(k-1)^{2}}{k} \\
& +\left(1.25 \times 10^{-4} \cdot s^{2}+1.9457 \times 10^{-6}\right) 14.849\left(\frac{\sqrt{1+s^{2}}}{s}\right)^{3} ; \\
& \quad \times \frac{\left(k^{2}-1\right)}{k}-0.2505 \frac{\sqrt{1+s^{2}}}{s} \ln k+\ln ^{2} k=0 \\
& S=7.938 \times 10^{-3} \frac{s}{\sqrt{1+s^{2}}}\left[\frac{117.809}{\sqrt{1+s^{2}}}-235 \frac{s}{1+s^{2}} \cdot \frac{\left(k^{2}-1\right)}{k^{2}}\right] .
\end{aligned}
$$

For hydrogen $\left(\theta=0,423 \times 10^{-3}\left(\mathrm{sec} \cdot \mathrm{m}^{-1}\right)\right.$

$6.7285 \times 10^{-4}\left(\frac{\sqrt{1+s^{2}}}{s}\right)^{3} \frac{(k-1)^{2}}{k}$

$$
+\left(1.25 \times 10^{-4} \cdot s^{2}+1.9457 \times 10^{-6}\right) 10.521\left(\frac{\sqrt{1+s^{2}}}{s}\right)^{3} \text {; }
$$

$$
\times \frac{\left(k^{2}-1\right)}{k}-0.1775 \frac{\sqrt{1+s^{2}}}{s} \ln k+\ln ^{2} k=0
$$

$$
\begin{aligned}
S= & 7.938 \times 10^{-3}-\frac{s}{\sqrt{1+s^{2}}} \\
& \times\left[\frac{117.809}{\sqrt{1+s^{2}}}-331.6784 \frac{s}{1+s^{2}} \cdot \frac{\left(k^{2}-1\right)}{k^{2}}\right]
\end{aligned}
$$

By varying angle $\varphi$ between $5^{\circ}<\varphi<60^{\circ}, k$ and $S$ take the values shown in Table 1. The functional dependence of $k$ and $S$ versus angle $\varphi$, is shown in Fig. 3 .

At this point, it can be concluded that for this specific pump, the best angle that ensures satisfactory compression ratio and satisfactory pumping speed, is that of $17.5^{\circ}$ where the compression for nitrogen is about 8 . Angle $\varphi$ can be varied between $10^{\circ}<\varphi \ll 40^{\circ}$, depending on our needs.

\section{Length calculation}

Finally let us calculate the proper length $X$ of the pump. Let $K$ be the total compression of the pump. Obviously the relation between the total compression of the pump and the compression of a single disc is

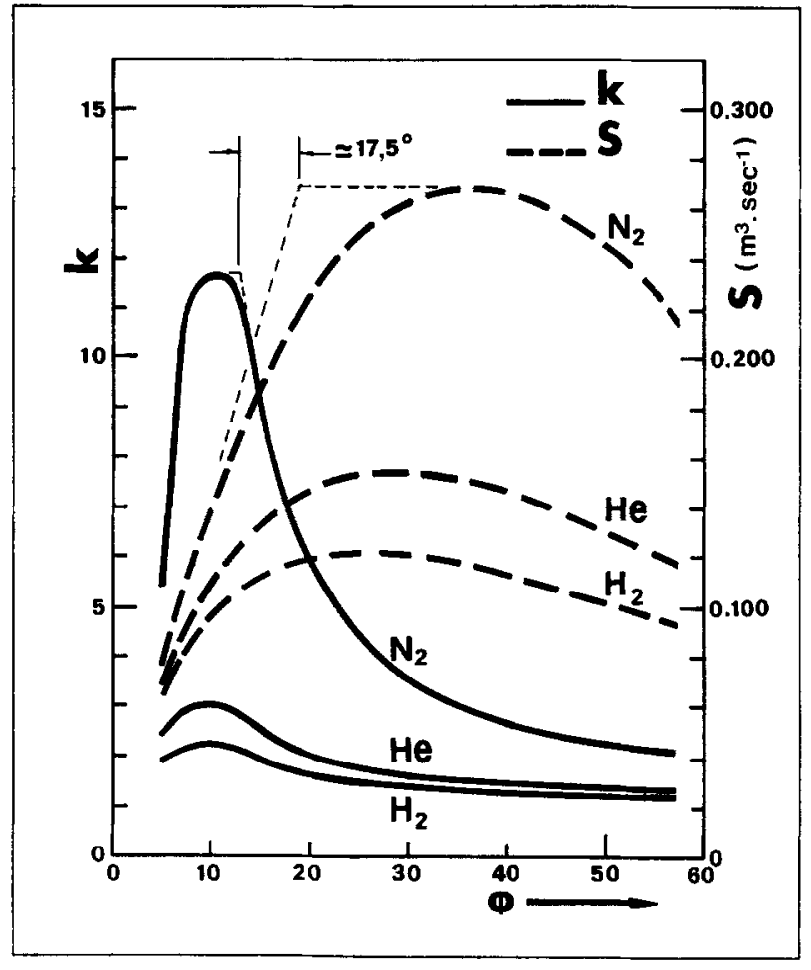

Figure 3. Compression ratio $k$ and pumping speed $S$ of the turbomolecular pump, versus angle $\phi$ for different gases $\left(\mathrm{N}_{2}, \mathrm{He}, \mathrm{H}_{2}\right)$ for a constant rotational speed.

$K=k^{2 T}$

where $T$ the number of stages of the pump. Solving for $T$ results $T=\frac{\log K}{2 \log k}$

Since, according to Fig. 2(a) the length $l_{0}$ for one stage is

$l_{0}=\frac{4 \pi r s}{v}$

the length for the pump will be

$X=l_{0} T-h^{\prime \prime}$.

Table 1. Variation of compression ratio $k$ and pumping speed $S$ of a turbomolecular pump, as a function of the blade angle $\varphi$ for $\mathrm{N}_{2}, \mathrm{He}, \mathrm{H}_{2}$

\begin{tabular}{rlllllll}
\hline & & \multicolumn{2}{l}{$\mathrm{N}_{2}$} & & $\mathrm{He}$ & \multicolumn{3}{l}{$\mathrm{H}_{2}$} & \\
& $S=\tan \varphi$ & \multicolumn{1}{l}{$k$} & $S\left(\mathrm{~m}^{3} \cdot \mathrm{sec}^{-1}\right)$ & $k$ & $S\left(\mathrm{~m}^{3} \cdot \mathrm{sec}^{-1}\right)$ & $k$ & $S\left(\mathrm{~m}^{3} \cdot \mathrm{sec}^{-1}\right)$ \\
\hline 5 & 0.087 & 5.36 & 0.076 & 2.40 & 0.069 & 1.92 & 0.064 \\
10 & 0.176 & 11.82 & 0.139 & 3.08 & 0.110 & 2.25 & 0.097 \\
15 & 0.268 & 8.85 & 0.189 & 2.41 & 0.134 & 1.87 & 0.112 \\
20 & 0.364 & 5.96 & 0.225 & 1.99 & 0.147 & 1.64 & 0.119 \\
25 & 0.466 & 4.42 & 0.250 & 1.77 & 0.153 & 1.50 & 0.122 \\
30 & 0.577 & 3.56 & 0.264 & 1.62 & 0.155 & 1.41 & 0.122 \\
40 & 0.840 & 2.69 & 0.268 & 1.46 & 0.147 & 1.31 & 0.113 \\
50 & 1.191 & 2.28 & 0.246 & 1.34 & 0.132 & 1.25 & 0.103 \\
60 & 1.732 & 2.04 & 0.204 & 1.31 & 0.113 & 1.21 & 0.092 \\
\hline
\end{tabular}


And by inserting into eqn (28) $T$ and $l_{0}$ values from eqns (26) and (27) we will have

$X=\frac{2 \pi r s}{v} \frac{\log K}{\log k}-h^{\prime \prime}$

We must note here that if (from eqn (26)) $T$ is a decimal number, we consider for $T$ value the next greatest integer.

Let us continue to consider the same example as we did before and let us calculate the length of the pump. If we take total compression $K=10^{\circ}$ and angle $\varphi=40^{\circ}$ then from Table 1 , for the case of Nitrogen, with $s=0.840$ and $k=2.69$, the pump will have 11 stages with a length $X=172.2 \times 10^{-3} \mathrm{~m}$.

\section{Conclusions}

From eqns (22) and (25) we see that for turbomolecular pumps, the compression ratio $k$ and the pumping speed $S$ depend on the values of geometrical parameters and especially on the angle $\varphi$ of the blades. But for a certain geometry, dimensions $h^{\prime}$ and $h^{\prime \prime}$ play a basic role if eqn (22) has to have a solution for $k \geqslant 1$. For this reason, the constructor has to pay attention to $h^{\prime}$ and $h^{\prime \prime}$ to be as small as possible.

We must also note that the optical opaqueness between the blades and along the rotational axis, suggested by Becker, although it improves the performance of a turbomolecular vacuum pump, is not adequate. The improvement needs something more. The mirror images of the rotor blades (shown in Fig. 2(a) with the bold dotted lines) must be in such a position relative to the stator blades, so that they form an Archemedes' helix with the latter. So if we want only the Becker suggestion to be valid, (see triangle $\mathrm{AB}^{\prime} \mathrm{C}^{\prime}$ of Fig. 4) we must have $h^{\prime \prime} \geqslant L s$. But if we also want our supplementary suggestion on to be valid, the choice is $h^{\prime \prime} \geqslant L s$

It is obvious from eqn (22) that the smaller $h^{\prime \prime}$ the larger the compression ratio $k$. So using our approach, the improvement is greater.

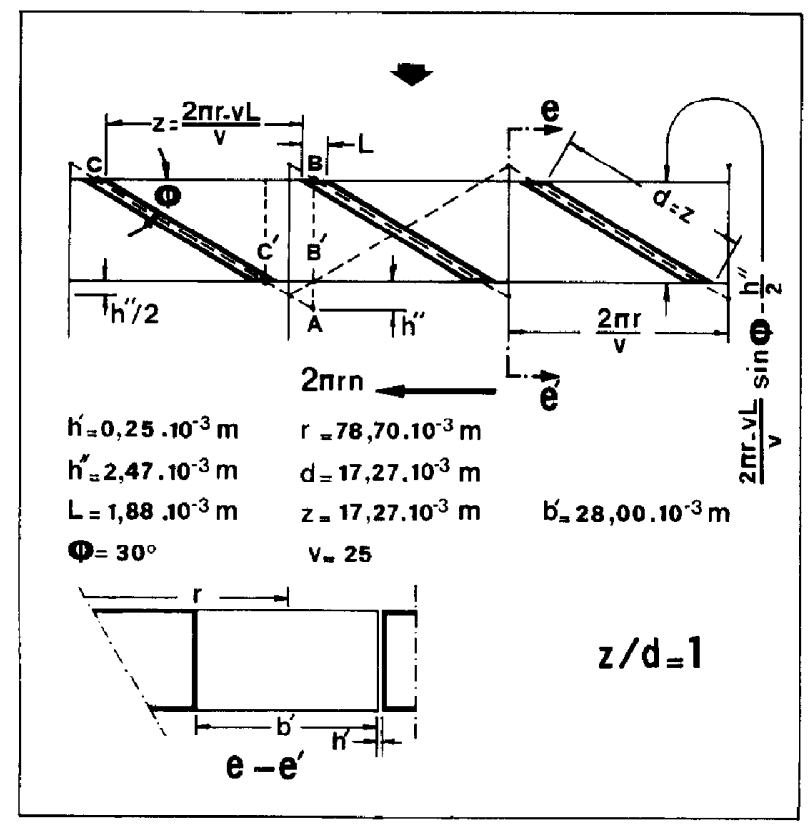

Figure 4. The single disc used by C. Kruger and A. Shapiro angle $\phi=30^{\circ}$ and the ratio $z / d=1$.
Referring to pumping speed $S$, [eqn (25)], it seems to have a similar behavior as in multi-groove molecular pumps when they operate in molecular state. ${ }^{6}$ Instead, pumping speed $S$ can be written as a sum of two terms $S_{1}$ and $S_{2}$, each one corresponding to a separate flow, the term

$S_{1}=\frac{\pi r n b^{\prime}(2 \pi r-v L) s}{1+s^{2}}$

which we call 'normal flow' is proportional to the rotational frequency $n\left[\mathrm{sec}^{-1}\right]$, while the term

$S_{2}=\frac{b^{\prime}(2 \pi r-v L)^{2} s^{2}\left(k^{2}-1\right)}{(8 \pi r) \theta \sqrt{\left(1+s^{2}\right)^{3}} k^{2}}$

which we call "inverse flow" depends on the nature of the gas to be puriped and on the rotational speed through the compression ratio $k$. In other words, for turbomolecular pumps the lighter the gas to be pumped ( $\theta$ becomes smaller) the smaller the pumping speed.

We must notice here that the solution of equations (22) and (25), for a turbomolecular pump, are satisfactory when the rotational speed $2 \pi r n \leqslant \sqrt{2 R T}$ (where $\sqrt{2 R T}$ is the most probable speed of the molecules). This limitation does not play any important role because rotational speeds greater than $\sqrt{2 R T}$ $\left(\mathrm{m} \cdot \mathrm{sec}^{-1}\right)$ for air, are of no interest in practice.

In Fig. 4 we see a description of a disc with blades of Kruger and Shapiro, ${ }^{2}$ where the dimensions $z$ and $d$ are equal and the angle of the blades is $30^{\circ}$. For air where the most probable speed $\sqrt{2 R T}=412\left(\mathrm{~m} \cdot \sec ^{-1}\right)$, the compression $\left(k^{\prime}=\Sigma_{12} / \Sigma_{21}\right)$ and the pumping speed ${ }^{8}\left(S^{\prime}=b^{\prime}(2 \pi r-v L) \sqrt{2 R T}\left(\Sigma_{12}-\Sigma_{21}\right) / 4\right)$ follow curve a of Fig. 5. If we want to construct a pump as described in Fig 2(a) and 2(b) this is possible when the distance $h^{\prime \prime}$ (between the rotor and stator discs) is calculated as follows.

Using identical triangles $\mathrm{ABC}$ and $\mathrm{AB}^{\prime} \mathrm{C}^{\prime}$ of Fig. 4, we have

$$
\frac{\frac{(2 \pi r-v L)}{v}(\sin \phi)+h^{\prime \prime}}{\frac{2 \pi r}{v}}=\frac{h^{\prime \prime}}{\frac{2 \pi r}{v}-\frac{(2 \pi r-v L)}{v} \cos \phi} .
$$

Solving for $h^{\prime \prime}$ we have

$$
h^{\prime \prime}=\frac{2 \pi r}{v} \tan \phi-\frac{2 \pi r-v L}{v} \sin \phi
$$

or (because $\tan \varphi=s$ ),

$h^{\prime \prime}=\left[2 \pi r-(2 \pi r-v L) \frac{1}{\sqrt{1+s^{2}}}\right] \frac{s}{v}$.

Using numerical values in eqn (30), according to Fig. 4,

$h^{\prime \prime}=2.47 \times 10^{-3} \mathrm{~m}$.

For any pump and for $h^{\prime}=h^{\prime \prime}=0$ (that is for a pump without losses), eqns (22) and (25) become respectively

$k=\exp (2 \pi r n) \Theta \frac{\sqrt{1+s^{2}}}{s}$

and 
Table 2. Values of compression ratio $k$ and pumping speed $S$, according eqns (32) and (33), and values of compression $k^{\prime}$ and pumping speed $S^{\prime}$ as they come from Fig. 4 from the thesis of Kruger' for a blade angle $\varphi=30^{\circ}$ and $z / d=1$

\begin{tabular}{lcllrl}
\hline$\frac{2 \pi r n}{\sqrt{2 R T}}$ & $n\left[\mathrm{sec}^{-1}\right]$ & $k^{\prime}$ & $\begin{array}{l}\text { Curves a } \\
S^{\prime}\left(\mathrm{m}^{3} \cdot \mathrm{sec}^{-1}\right)\end{array}$ & $k$ & $\begin{array}{l}\text { Curves b } \\
S\left(\mathrm{~m}^{3} \cdot \mathrm{sec}^{-1}\right)\end{array}$ \\
\hline 0.25 & 208.3 & 1.43 & 0.161 & 1.44 & 0.163 \\
0.50 & 416.6 & 1.99 & 0.336 & 2.08 & 0.362 \\
0.75 & 624.9 & 2.95 & 0.509 & 4.01 & 0.584 \\
1.00 & 833.2 & 4.15 & 0.646 & 6.26 & 0.825 \\
1.25 & 1041.5 & 5.64 & 0.757 & 9.03 & 1.078 \\
1.50 & 1249.8 & 7.20 & 0.840 & 13.04 & 1.605 \\
1.75 & 1458.1 & 8.46 & 0.897 & 18.81 & 1.875 \\
2.00 & 1666.4 & 9.08 & 0.914 & & \\
\hline
\end{tabular}

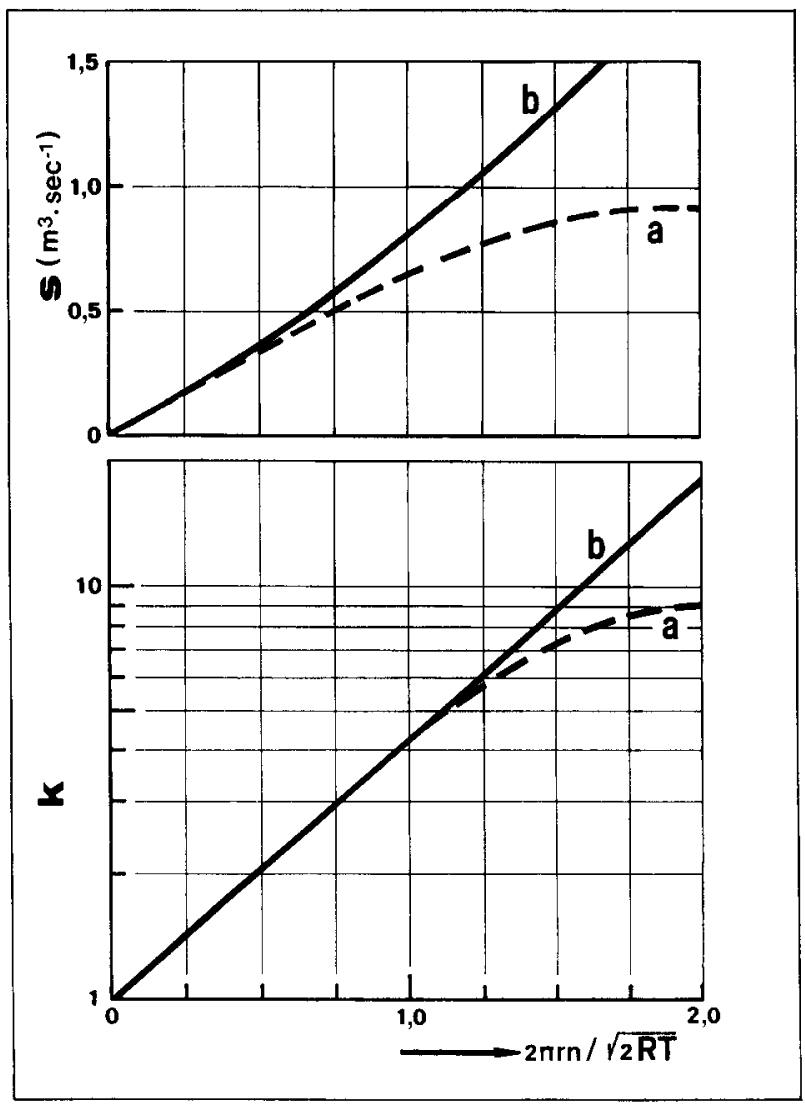

Figure 5. Calculated results for $k$ and $S$ for a single disc with $\phi=30^{\circ}$ and $z / d=1$.

(i) By C. Kruger and A. Shapiro (curves a).

(ii) By the present paper (curves b).

$$
S=b^{\prime} 2(\pi r)^{2} n-\frac{s}{\sqrt{1+s^{2}}}\left[1-\frac{s}{4 \pi r \Theta n \sqrt{1+s^{2}}} \cdot \begin{array}{c}
(k-1) \\
k
\end{array}\right]
$$

From these last two equations and the dimensions of Fig. 4, for air with $\theta=1.61 \times 10^{-2}\left(\mathrm{~m} \cdot \mathrm{sec}^{-1}\right)$, we obtain Table 2 and finally curve b of Fig. 5 .

By this way and from Fig. 5, it is possible to compare the results of our study with those of Kruger and Shapiro. For velocities smaller than $\sqrt{2 R T}$, from curves $a$ and $b$ we see the same results for the compression ratio $\mathrm{k}$, and a deviation of about $15 \%$ for the greater values of pumping speed.

We believe that our treatment for a turbomolecular pump is simple to use because it gives (to any constructor) the possibility of calculating the pump according the parameters and specifications preferred, from equations (32) and (33) using only a pocket calculator, in other words, knowing the size of the pump and its performance through the losses.

\section{References}

'W Becker, eine neue Molekularpumpe, Vakuum Technik, Jahrgang 7, 149. Heft 7, (1958). Proc. 1st Int. Vac. Congress, Namur (1958).

${ }^{2} \mathrm{C}$ Kruger and A Shapiro, Vacuum pumping with a bladed axial-flow turbomachine 7 Nat. Symp. Vac. Techn. Transact., 6-12, 1960.

${ }^{3} \mathrm{~W}$ Becker, The turbomolecular pump, its design, operation and theory. Vacuum, 16, (1966), 625.

${ }^{4}$ W Gaede, Die Molekular luftpumpe Ann. Physik, 41, 342, (1913).

${ }^{5} \mathrm{~W}$ Becker and $\mathrm{W}$ Nesseldreher, New high performance dises for turbomolecular pump. Vacuum, 26, 277, 1976.

${ }^{\circ} \mathrm{C}$ N Panos, A G Antoniou and S E Valamonte, The helicoid multigroove vacuum pump in both viscous and molecular states. Vacuum 45 , (1994) 841-7.

'W Becker, Die Turbomolekularpumpe. Vakuum Technik, Jahrgang 15, Heft 9, 211, 254 (1966).

${ }^{8} \mathrm{~J} \mathrm{G}$ Chu and Z Y Hua, The statistical theory of turbomolecular pumps. J. Vac. Sci. Technol., 20, (1982) 1101.

${ }^{9} \mathrm{C} \mathrm{H}$ Kruger, The axial-flow compressor in the free molecule range Ph.D. Thesis, Dept of Mech. Eng., M.I.T., Cambridge, Mass. (1960). 